\title{
A New Vision for the National Institutes of Health
}

\author{
Elias A. Zerhouni, MD* \\ National Institutes of Health, 1 Center Drive, Bethesda, MD 20892, USA
}

The National Institutes of Health (NIH) has grown from a one-room bacteriology laboratory in 1887 into a multibillion-dollar medical research enterprise. While the $\mathrm{NIH}$ has remained true to its original mission-pursuing scientific knowledge to improve people's health-almost everything else about it has changed.

Today, NIH comprises 27 Institutes and Centers that sponsor medical research in areas ranging from cancer to diabetes and from genomics to alternative medicine.

With the dramatic increases in scientific knowledge as well as the significant changes in diagnosis, treatment, prevention, translation, and delivery of care, researchers must continue to meet the challenges of tomorrow by adopting new strategies today.

The NIH is pioneering a vision that will determine what the agency should be and should do in this new "biomedical century." We are focusing our research energies in three specific areas: exploiting new pathways of discovery; encouraging the formation of interdisciplinary teams of the future; and reengineering the clinical research enterprise.

In the first area, we know that scientific research is cost-effective because it saves lives and money in both the short and the long run. Witness our successes in working with AIDS, coronary heart disease, and cancer. We also know that health care costs are continuing to spiral upward, partly due to the aging population which is placing more and more demands on the system.

To keep up with the projected increases in costs, the research enterprise must not only accelerate the pace of discovery, but also apply its research results in a timely manner. I believe this is one of the greatest challenges we face in this biomedical century.

In the second area, discoveries in human biology are occurring at unprecedented speed, presenting opportunities in medicine that we could only dream about just a few years ago. But these opportunities require a shift in how medical research is conducted and funded.

While investigator-initiated projects are the mainstay of NIH-supported research, increasingly these projects involve larger multidisciplinary teams of scientists. These teams might include specialists in disciplines outside biology, such as computer science, imaging, chemistry, math- ematics, and informatics. Such multidisciplinary science teams are the wave of the future.

I believe that we need to break down the walls that exist between scientific disciplines, inside and outside NIH. We need to foster the growth of interdisciplinary teams in order to maximize the enormous potential of research to improve our lives.

For its part, NIH is working to meet this challenge by filling leadership positions at its Institutes and Centers with outstanding scientists who have experience with this new paradigm.

In the third area, we need to reengineer the national clinical research enterprise in order to most effectively translate our discoveries into clinical reality. The list of initiatives to undertake is long, but necessary. It includes supporting multidisciplinary clinical research training career paths, introducing innovations in trial design, stimulating translational research, building clinical resources like tissue banks, developing large clinical research networks, and reducing regulatory hurdles. We must also explore a standard clinical research informatics strategy, which will permit the formation of Nation-wide "communities" of clinical researchers made up of academic researchers, qualified community physicians, and patient groups.

Alongside our dedicated efforts to pioneer a new vision for NIH is the continuing need to attract the best and brightest researchers. We in the scientific community realize the need to cultivate and nurture minority talent if scientific research is to remain a viable enterprise. Currently, we are experiencing difficulty in retaining researchers from the minority communities, especially through the doctoral levels.

We must identify and attract students with potential early on and not let them "fall through the cracks" of academia. We need to consider novel approaches to encourage minority students to remain in mainstream science. Providing students with knowledgeable and supportive mentors and role models could serve as an enticement.

$\mathrm{NIH}$ has been a leader in supporting minority students throughout their education, with numerous programs and policies in place to increase the number of 
minorities in research careers. As we take on the challenge of conducting scientific research in the 21st century by pursuing the NIH vision, it is absolutely critical that we find ways to recruit and retain more women and minorities in scientific careers.

Elias A. Zerhouni, MD

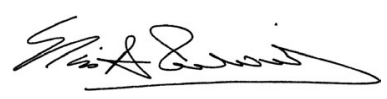

Elias A. Zerhouni began his tenure as the 15th Director of the National Institutes of Health on May 20, 2002. Dr Zerhouni initiated the creation of a new research vision for the NIH which focuses the attention of the biomedical research community on new pathways of discovery, research teams for the future, and the reengineering of the clinical re-

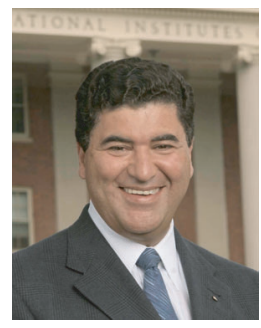
search enterprise. Among his noteworthy achievements since becoming Director, Dr Zerhouni has named directors for three institutes - the National Institute of Mental Health (Thomas R. Insel, MD), the National Institute on Alcohol Abuse and Alcoholism (Ting-Kai Li, MD), and the National Institute on Drug Abuse (Nora D. Volkow, MD). He also named a new NIH Deputy Director (Raynard S. Kington, MD, PhD) and a new director of the Office of Technology Transfer (Mark L. Rohrbaugh, $\mathrm{PhD}$, JD) He has also overseen the completion of the doubling of the NIH budget during the last five years. His research in imaging led to advances in Computerized Axial Tomography (CAT scanning) and Magnetic Resonance Imaging (MRI) that resulted in 157 peer reviewed publications and 8 patents.

*E-mail: director@NIH.gov 

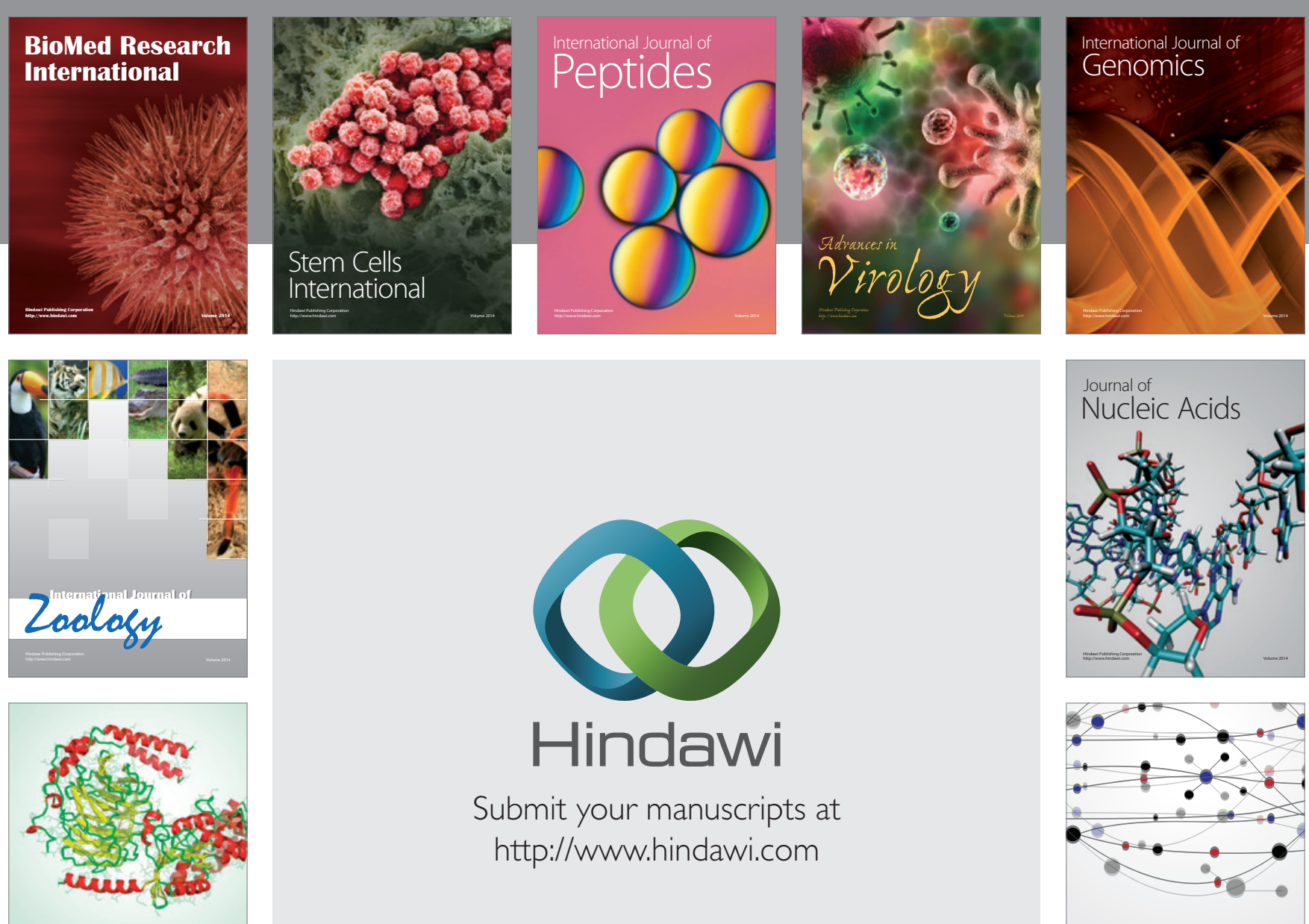

Submit your manuscripts at

http://www.hindawi.com

Signal ${ }^{\text {Jumal }}$ Transduction
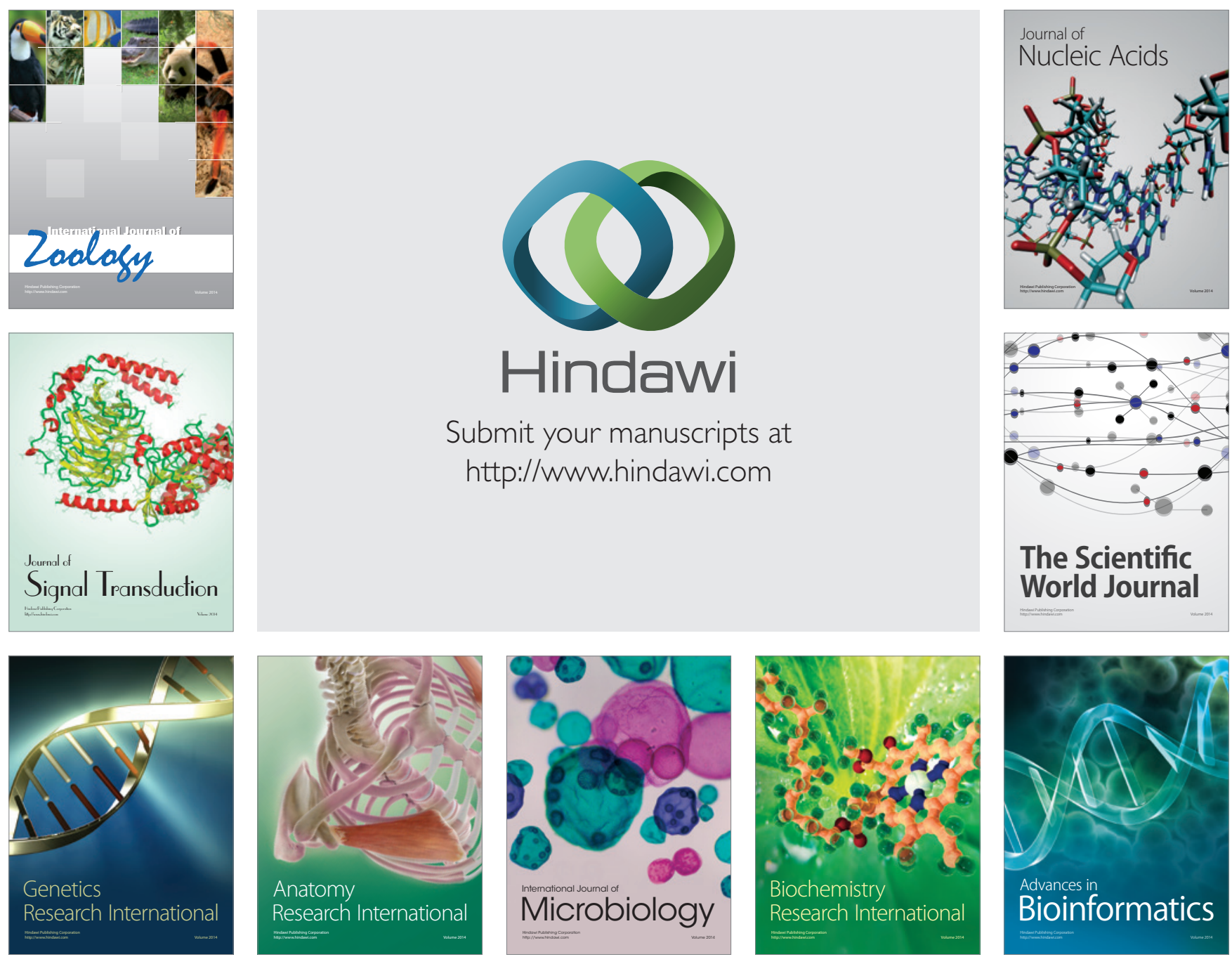

The Scientific World Journal
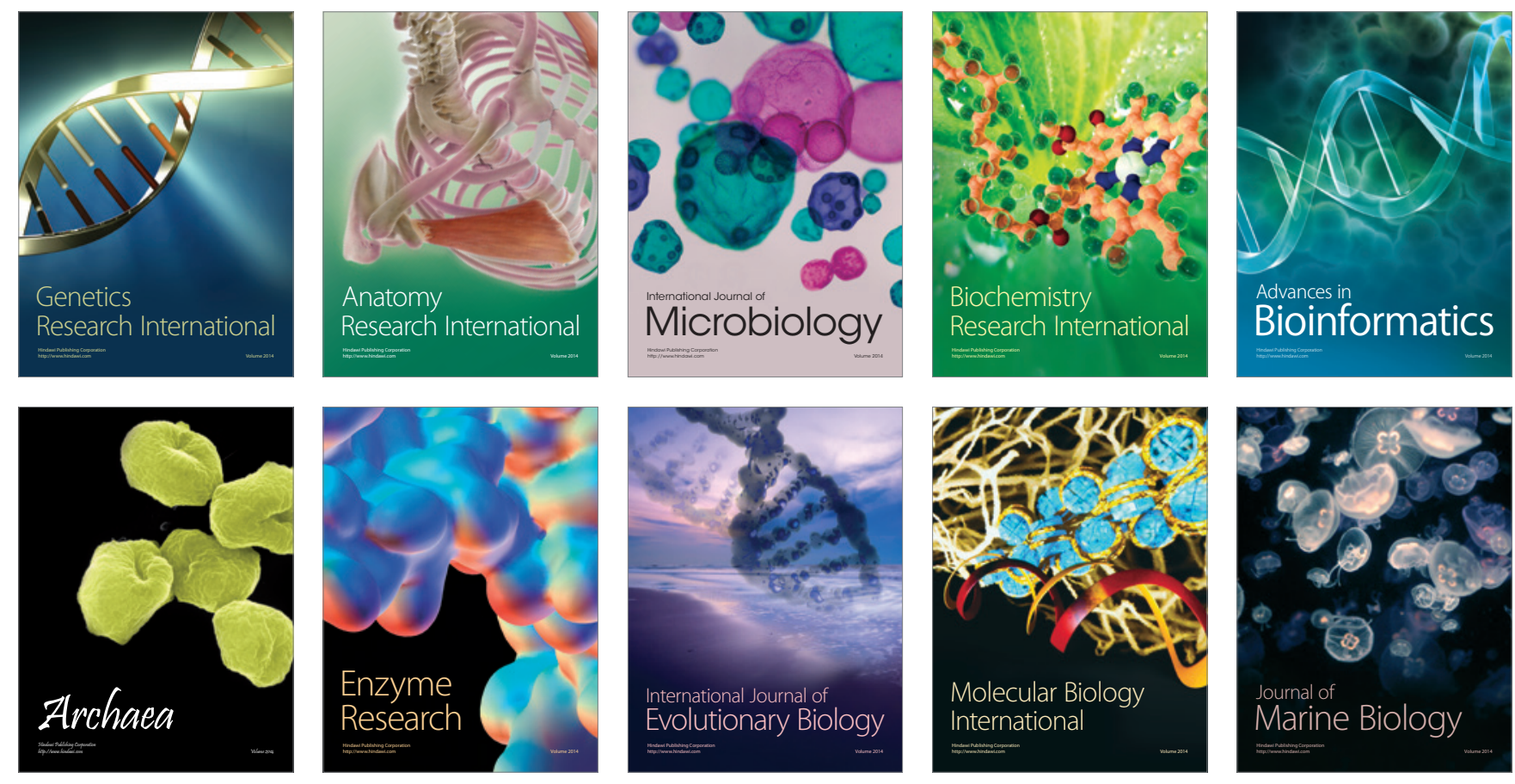